\title{
Network-based cancer genomic data integration for pattern discovery
}

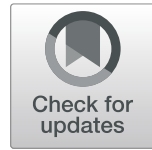

Fangfang Zhu' ${ }^{1,2 \dagger}$, Jiang $\mathrm{Li}^{2 *}$, Juan $\mathrm{Liu}^{3 *}$ and Wenwen Min $4,5^{*}+$ (D)

From Fifteenth International Conference on Intelligent Computing (ICIC 2019)

Nanchang, China. 3-6 August 2019

\begin{abstract}
Background: Since genes involved in the same biological modules usually present correlated expression profiles, lots of computational methods have been proposed to identify gene functional modules based on the expression profiles data. Recently, Sparse Singular Value Decomposition (SSVD) method has been proposed to bicluster gene expression data to identify gene modules. However, this model can only handle the gene expression data where no gene interaction information is integrated. Ignoring the prior gene interaction information may produce the identified gene modules hard to be biologically interpreted.

Results: In this paper, we develop a Sparse Network-regularized SVD (SNSVD) method that integrates a prior gene interaction network from a protein protein interaction network and gene expression data to identify underlying gene functional modules. The results on a set of simulated data show that SNSVD is more effective than the traditional SVD-based methods. The further experiment results on real cancer genomic data show that most co-expressed modules are not only significantly enriched on GO/KEGG pathways, but also correspond to dense sub-networks in the prior gene interaction network. Besides, we also use our method to identify ten differentially co-expressed miRNA-gene modules by integrating matched miRNA and mRNA expression data of breast cancer from The Cancer Genome Atlas (TCGA). Several important breast cancer related miRNA-gene modules are discovered.

Conclusions: All the results demonstrate that SNSVD can overcome the drawbacks of SSVD and capture more biologically relevant functional modules by incorporating a prior gene interaction network. These identified functional modules may provide a new perspective to understand the diagnostics, occurrence and progression of cancer.
\end{abstract}

Keywords: Gene co-expression analysis, Differentially co-expression analysis, Gene interaction network, Sparse SVD, Structured sparse learning

\footnotetext{
*Correspondence: li66001@163.com; liujuan@whu.edu.cn; mww@whu.edu.cn

${ }^{\dagger}$ Fangfang Zhu and Wenwen Min contriubted equally to this work.

${ }^{4}$ School of Mathematics and Computer Science, Jiangxi Science and

Technology Normal University, 330038 Nanchang, China

${ }^{5}$ Information School, Yunnan University, 650091 Kunming, China

Full list of author information is available at the end of the article
}

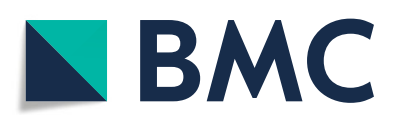

(c) The Author(s). 2021 Open Access This article is licensed under a Creative Commons Attribution 4.0 International License, which permits use, sharing, adaptation, distribution and reproduction in any medium or format, as long as you give appropriate credit to the original author(s) and the source, provide a link to the Creative Commons licence, and indicate if changes were made. The images or other third party material in this article are included in the article's Creative Commons licence, unless indicated otherwise in a credit line to the material. If material is not included in the article's Creative Commons licence and your intended use is not permitted by statutory regulation or exceeds the permitted use, you will need to obtain permission directly from the copyright holder. To view a copy of this licence, visit http://creativecommons.org/licenses/by/4.0/. The Creative Commons Public Domain Dedication waiver (http://creativecommons.org/publicdomain/zero/1.0/) applies to the data made available in this article, unless otherwise stated in a credit line to the data. 


\section{Background}

With the rapid development of (single-cell) RNA-Seq and microarray technologies, huge number of cancer genomic data have been generated [1-3]. The data provide some new opportunities to study on the gene cooperative mechanisms [4-8]. Based on the hypothesis that genes with similar functions may show similar expression patterns, clustering techniques have been used to identify co-expressed gene sets in which genes present similar expression patterns across all samples. However, these traditional clustering techniques face with the limitation that some genes can co-regulate across some samples rather than all samples in the real biological systems [9]. Therefore, many biclustering methods $[4,10-13]$ are proposed to discover some co-expressed gene sets in which genes present similar expression patterns across some samples.

Recently, several Sparse Singular Value Decomposition (SSVD) based methods have been proposed for biclustering gene expression data to discover gene functional modules (biclusters) [14], such as ALSVD [4], LOSVD [15], and so on. However, most of them ignore the prior gene interaction network knowledge from a protein protein interaction (PPI) network, whereas such information is very useful to improve biological interpretability of discovered gene modules [16-18]. The PPI network has been used in many biological applications for accurate discovery or better biological interpretability [16, 19-22]. However, as far as we know, there is very little work to incorporate the gene interaction network knowledge from PPI network into the bi-clustering framework. To address it, we integrate the gene network in the SSVD model for biclustering gene expression [23].

In this paper, we develop a sparse network-regularized SVD (SNSVD) model to identify gene functional modules by integrating gene expression data and a prior gene interaction network from a PPI network (Fig. 1). To ensure the discovered gene modules in which genes are co-expressed and densely connected in the prior PPI network, we introduce a sparse network-regularized penalty [20] in the model. Compared with the traditional regularized penalties (e.g., LASSO [24]), the sparse network-regularized penalty can make the biclustering process tend to select correlated and interacted genes for enhancing biological interpretability of gene modules. We present an alternating iterative algorithm to solve the SNSVD model. We evaluate the performance of SNSVD using a set of artificial data sets and gene expression data from the Cancer Genome Project (CGP) [3], and compare its performance with other representative SSVD methods. We investigate the functionality of these identified modules from multiple perspectives. The results show that SNSVD can identify more biologically relevant gene sets and improve their biological interpretations.
Additionally, we present a framework based on SNSVD for analyzing matched miRNA and mRNA expression data to identify differentially co-expressed miRNAgene modules. Extensive results when applying onto TCGA breast cancer data demonstrate that the identified miRNA-gene modules provide a new perspective for diagnosis and discrimination between two status of breast cancer.

\section{Results}

\section{Simulation study}

We evaluated the performance of SNSVD on the simulated data by comparing it with other sparse SVD based methods including LOSVD [15], ALSVD [4] and SCADSVD $[4,25]$. Without loss of generality, we define a rank-one true signal matrix as $\boldsymbol{u} \boldsymbol{v}^{T}$ where $\boldsymbol{u}$ and $\boldsymbol{v}$ are vectors of size $p \times 1$ and $n \times 1$, respectively. The observed matrix is defined as $\boldsymbol{X}=\boldsymbol{u} \boldsymbol{v}^{T}+\gamma \boldsymbol{\epsilon}$, where $\boldsymbol{\epsilon}$ is a noise matrix each element in which is randomly sampled from a standard normal distribution and $\gamma$ is a nonnegative parameter to control the signal-to-noise ratio (SNR).

To generate the simulated data, we first generated two sparse singular vectors $\boldsymbol{u}$ and $\boldsymbol{v}$ with $p=200, n=100$ whose first 50 elements equal to 1 (non-zeros), and the remaining ones are zeros. Then we created a series of observation matrices $X$ for each $\gamma$ ranging from 0.02 to 0.06 in steps of 0.005 . In addition, we created a prior "PPI" network for row variables of $\boldsymbol{X}$, where any two nodes in first 50 vertices are connected with probability $p_{11}=0.3$, and remaining ones are connected with probability $p_{12}=$ 0.1 . For each $\gamma$, we generated 50 different noise matrices $\boldsymbol{\epsilon}$ to got 50 observed matrices $X$ for testing. The average sensitivity, specificity and accuracy of $\boldsymbol{u}$ (or $\boldsymbol{v}$ ) on the 50 matrices $X$ were calculated. Moreover, we set $\sigma=0.5$ according to 5 -fold cross validation test, and forced $\boldsymbol{u}$ and $\boldsymbol{v}$ to contain 50 non-zero elements with same sparsity level for each method by tuning the parameters so that the results of different methods are comparable. The average sensitivities, specificities and accuracies of $\boldsymbol{u}$ (or $v)$ with different $\gamma$ were compared in Fig. 2. We found that the performance of our proposed method (SNSVD) is superior to that of other methods. The results illustrate that SNSVD model can enhance the power of variable selection by integrating the prior network knowledge.

\section{Application to the CGP gene expression data sets}

We further investigated the performance of SNSVD by using the gene expression data with 641 cell lines including diverse cancer types and tissues from the Cancer Genome Project (CGP) (http://www.cancerrxgene.org/ downloads) [3], and a PPI network from the PathwayCommons database [26]. In total, there are 13,321 genes and 262,462 interactions in the PPI network. The 641 cell lines are from 16 tissues or 52 cancer types in the CGP 


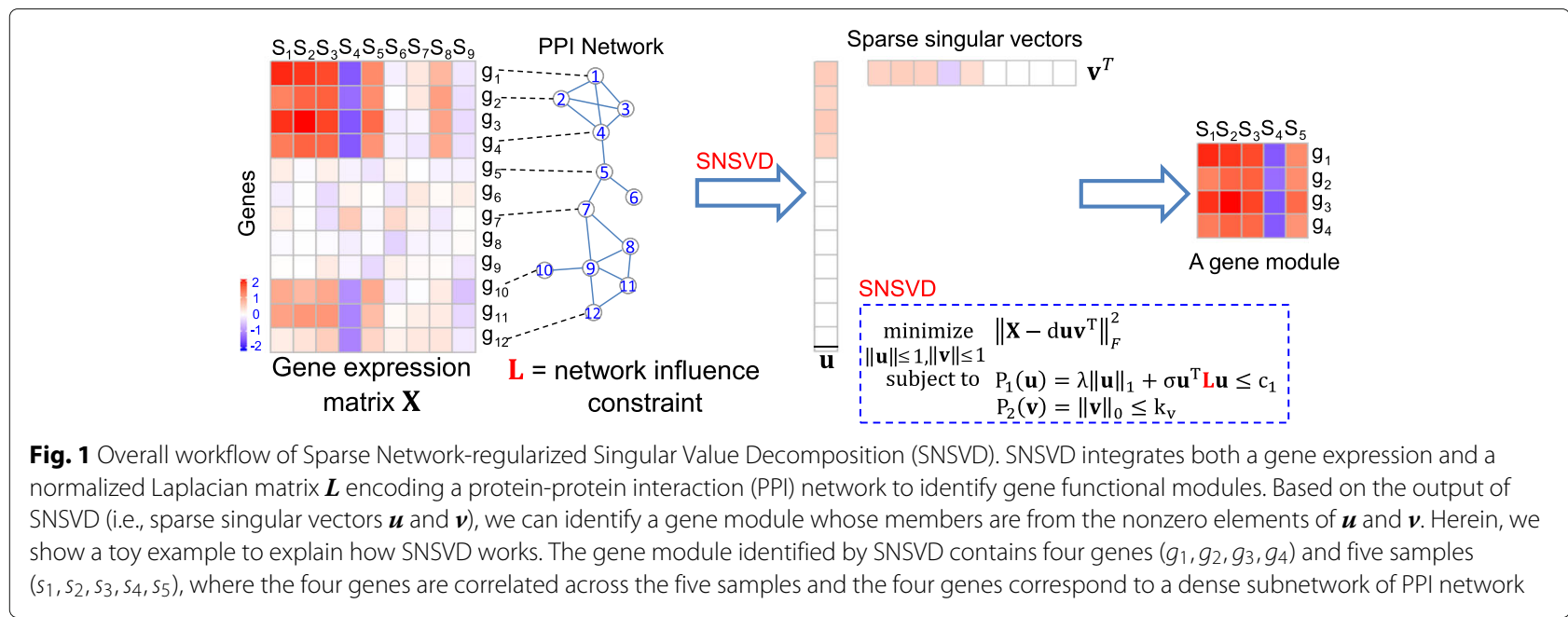

data, where a tissue type contains about 40 cell lines and a cancer type contains about 12 cell lines.

\section{Identifying functional modules}

We set $\sigma=100$ according to 5 -fold cross validation test, and set $k_{v}=50$ (control the sample sparsity). In addition, we also selected a suitable $\lambda$ to force the estimated $\boldsymbol{u}$ only containing 200 nonzero elements (control the gene sparsity). Using Algorithm 3, we identified the first 40 pairs of singular vectors $\left\{\left(\boldsymbol{u}_{1}, \boldsymbol{v}_{1}\right), \cdots,\left(\boldsymbol{u}_{40}, \boldsymbol{v}_{40}\right)\right\}$. Let $\boldsymbol{U}=\left[\boldsymbol{u}_{1} ; \cdots ; \boldsymbol{u}_{40}\right]$ and $\boldsymbol{V}=\left[\boldsymbol{v}_{1} ; \cdots ; \boldsymbol{v}_{40}\right]$, where the $i$ th column of $\boldsymbol{U}$ and $\boldsymbol{V}$ correspond to the $i$ th pair of sparse

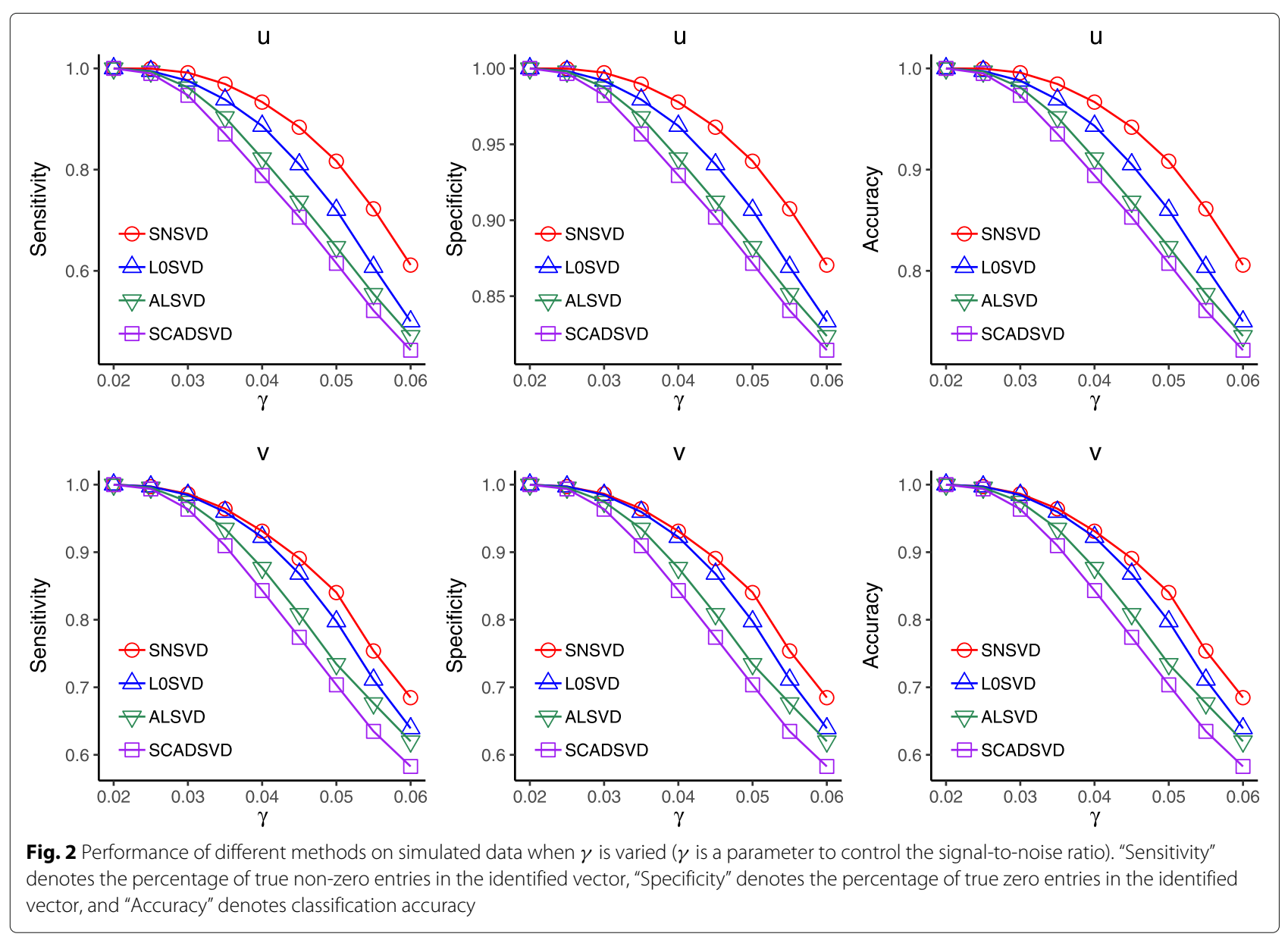



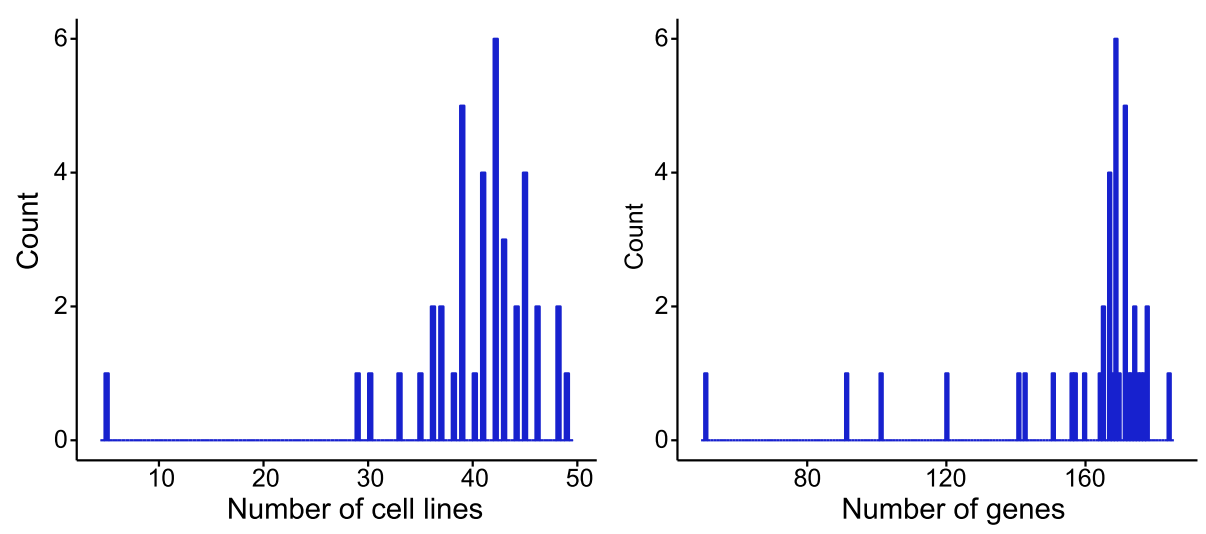

Fig. 3 Distribution of the number of samples (i.e., cell lines) and genes from the identified modules by SNSVD on the CGP data

singular vectors. To reduce the false positive cases, we first calculated absolute z-score for each column of $\boldsymbol{U}$ (or $\boldsymbol{V}$ ) according to Eq. (1). For each non-zero $x_{i j}$, we define the following formula:

$$
z_{i j}=\frac{|| x_{i j}\left|-\mu_{j}\right|}{\sigma_{j}}
$$

where $x_{i j}$ is $i$-th element in $\boldsymbol{u}_{j}\left(\right.$ or $\left.\boldsymbol{v}_{j}\right), \mu_{j}$ is the average value of all non-zero elements in $\boldsymbol{u}_{j}$ (or $\boldsymbol{v}_{j}$ ), and $\sigma_{j}$ is their standard deviation. If $z_{i j}$ is greater than a given threshold, the corresponding gene (or sample) is then selected into the module $j$. Herein, we obtained 40 gene functional modules with 160 genes and 40 samples in average (Fig. 3).

\section{Functional analysis of the genes in modules}

Firstly, we investigated whether the genes within the same modules are significantly co-expressed by calculating the modularity score in Eq. (17), the result showed that all identified modules were statistically significant with $p$ value $<0.01$ by using one-sided Wilcoxon signed rank test (Fig. 4).

Secondly, we also investigated whether the genes within the same modules are connected with each other in the prior PPI network via the gene-gene interaction enrichment score. The result showed that $57 \%$ of the 40 modules were significantly inter-connected with each other in the PPI network, illustrating that our method tend to cluster genes interacting with each other.

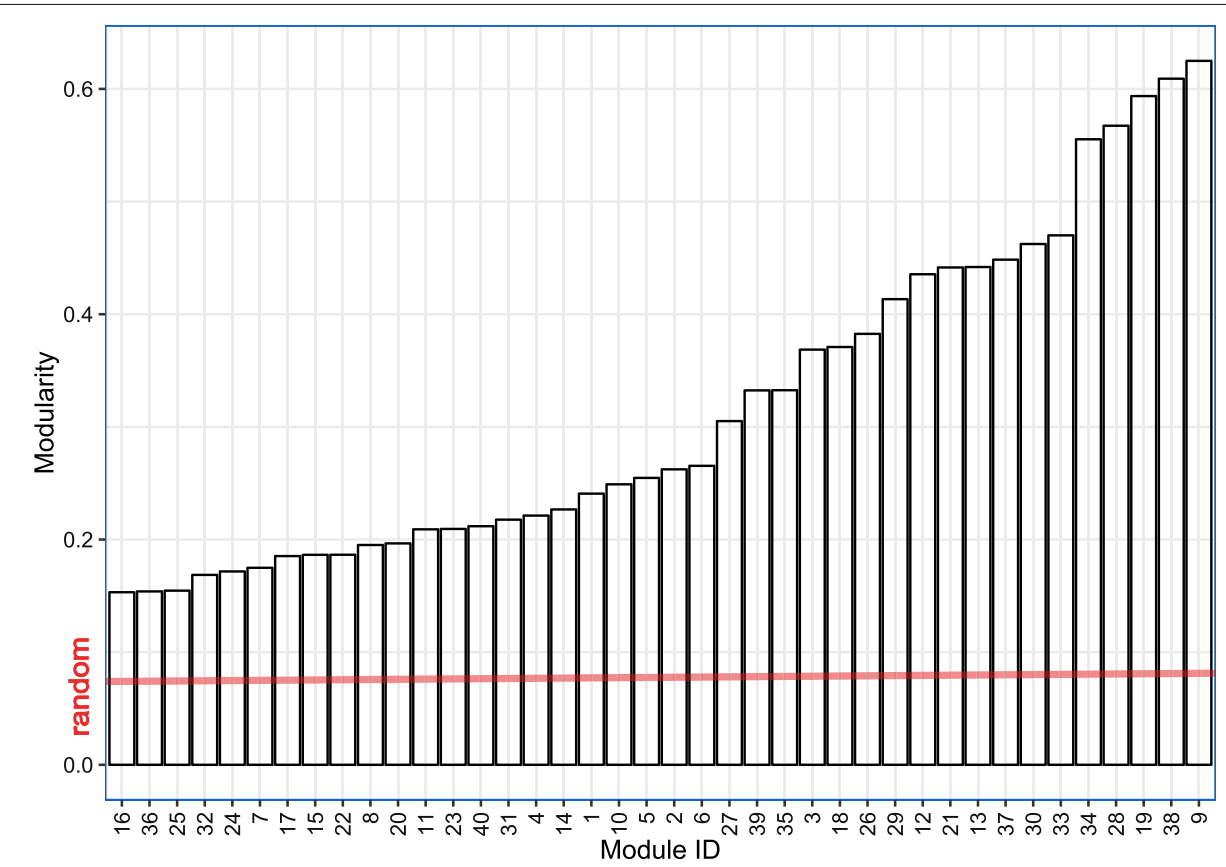

Fig. 4 The modularity scores of the identified modules by SNSVD on the CGP data. The red line in plot denotes an average modularity score of randomized modules (gene sets) 
Finally, we also checked the biological relevance of all the identified gene modules using gene functional enrichment analysis via DAVID online web tool [27]. By selecting the GO BP (Gene Ontology Biological Process) and KEGG pathways with Benjamini-Hochberg adjusted $p$-values < 0.05 as significant ones, we obtained 766 significant GO BP pathways and 70 significant KEGG pathways. By statistically, $62.5 \%$ modules are significantly related with at least one GO BP pathways and $42.5 \%$ modules are significantly related with at least one KEGG pathways.

\section{Functional analysis of the samples in modules}

To evaluate the subtype-specific of samples in the identified modules, we computed the overlapping significance level of between module-samples and cancer/tissue specific samples. For each gene module, we first collected a sample set from the module. We then computed the overlapping significance levels between the sample set and any one tissue-sample set using the right hypergeometric test (Fig. 5A), and the overlapping significance levels between

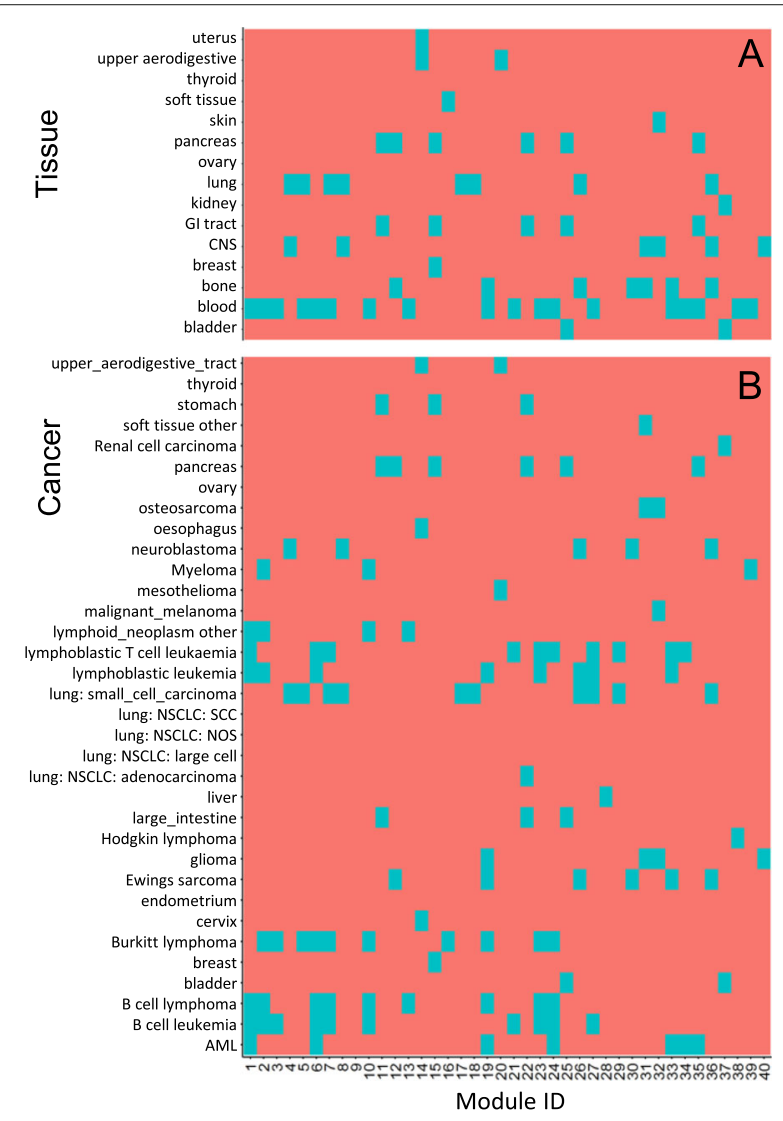

Fig. 5 These identified gene modules by SNSVD are subtype-specific related to some tissues or cancer types. $\mathbf{A}$ is a heatmap in term of different tissues. $\mathbf{B}$ is a heatmap in term of different cancer types. Note that each blue square in the two heatmaps corresponds to a significance overlapping relationship (Hypergeometric test, $p<0.05$ ) the sample set and any one cancer-sample set (Fig. 5B). We found that most of the identified gene modules can be seen as subtype-specific gene functional modules, which provide insights into the mechanisms of the relationship between different tissues and cancers.

Additionally, we also found that the cancer/tissue types of some modules are consistent with their corresponding functional pathways. Some examples are listed in Table 1 (See also Fig. 5). For example, module 1 contains 47 cell lines significantly overlapping with blood tissue and some blood-related cancers (e.g., AML, B cell leukemia, B cell lymphoma, lymphoblastic leukemia, lymphoblastic $\mathrm{T}$ cell leukaemia, lymphoid_neoplasm other), while the top enriched GO/KEGG pathways of 174 genes in module 1 are related to the immune system. Some previous work have reported that the development of blood-related cancers are associated with immune pathway abnormalities [28, 29]. Similarly, these samples in module 2 are also significantly related with some blood-related cancers (B cell leukemia, B cell lymphoma, Burkitt lymphoma, lymphoblastic leukemia, and lymphoid_neoplasm other), while some genes in which are significantly enriched in some immune-related pathways. These samples in module 4 are significantly related with central nervous system (CNS), while some genes in which are significantly enriched in nervous system related GO/KEGG pathways.

Finally, we also evaluated whether the identified 40 modules are greatly overlapped. Since each module contains a gene set and a sample set. To assess the overlapping relationship between two different modules. For any two gene modules, we computed the overlapping significance level $p_{1}$ and $p_{2}$ between their gene sets and sample sets respectively by using the right-tailed hypergeometric test. If $p_{1}<0.05$ and $p_{2}<0.05$, then we considered that the two modules are significant overlapped. Among all 780 module-module pairs for the identified 40 modules, we found that only 17 out of the 780 module-module pairs are significantly overlapped (Fig. 6), showing that our method can find diverse functional modules.

\section{Comparison with sparse SVD on the CGP gene expression data sets}

Since LOSVD have shown good performances in simulation study compared to other sparse SVD methods, we compared it with our method to further illustrate the importance of integrating the PPI network. To this end, we also identified 40 gene modules on the CGP data by using LOSVD and Fig. 7 shows the comparing results. We found that the interaction enrichment scores of the identified modules by SNSVD were significantly higher than that by LOSVD (one-sided Wilcoxon signed rank test $p$-value $<0.01)$ (Fig. 7A). These results demonstrate that SNSVD can find more tightly connected genes 
Table 1 The first five enriched GO/KEGG pathways of top ten modules identified by SNSVD on the CGP data where "P-value" denotes Benjamini-Hochberg adjusted $P$-value

\begin{tabular}{|c|c|c|}
\hline Module & Enriched GO/KEGG pathways & $P$-value \\
\hline 1 & GO:0006952 defense response & $3.08 \mathrm{e}-12$ \\
\hline 1 & GO:0001775 cell activation & $1.11 \mathrm{e}-10$ \\
\hline 1 & GO:0045321 leukocyte activation & $2.55 e-10$ \\
\hline 1 & GO:0006955 immune response & $7.34 \mathrm{e}-10$ \\
\hline 1 & GO:0042110 T cell activation & $1.42 \mathrm{e}-07$ \\
\hline 2 & GO:0006955 immune response & $4.03 e-12$ \\
\hline 2 & GO:0006414 translational elongation & $1.28 \mathrm{e}-08$ \\
\hline 2 & hsa03010:Ribosome & $2.96 \mathrm{e}-08$ \\
\hline 2 & hsa04662:B cell receptor signaling pathway & $4.31 \mathrm{e}-08$ \\
\hline 2 & GO:0001775 cell activation & $2.00 \mathrm{e}-07$ \\
\hline 3 & GO:0006414 translational elongation & $2.11 \mathrm{e}-86$ \\
\hline 3 & hsa03010:Ribosome & $3.28 \mathrm{e}-81$ \\
\hline 3 & GO:0006412 translation & $6.62 \mathrm{e}-57$ \\
\hline 3 & $\begin{array}{l}\text { GO:0042273 ribosomal large subunit } \\
\text { biogenesis }\end{array}$ & $3.27 e-04$ \\
\hline 3 & GO:0042254 ribosome biogenesis & $2.38 \mathrm{e}-03$ \\
\hline 4 & GO:0006836 neurotransmitter transport & $8.83 e-06$ \\
\hline 4 & GO:0030182 neuron differentiation & $3.50 \mathrm{e}-03$ \\
\hline 4 & GO:0007269〜neurotransmitter secretion & $6.71 e-03$ \\
\hline 4 & GO:0050767 regulation of neurogenesis & $1.79 \mathrm{e}-02$ \\
\hline 4 & $\begin{array}{l}\text { GO:0048667 cell morphogenesis involved in } \\
\text { neuron diff. }\end{array}$ & $1.83 e-02$ \\
\hline 6 & GO:0042110 T cell activation & $8.05 e-11$ \\
\hline 6 & hsa04660:T cell receptor signaling pathway & $3.35 e-09$ \\
\hline 6 & GO:0045321 Neukocyte activation & $1.08 \mathrm{e}-08$ \\
\hline 6 & GO:0001775 cell activation & $1.43 e-08$ \\
\hline 6 & GO:0046649 lymphocyte activation & $2.44 \mathrm{e}-08$ \\
\hline 7 & GO:0051276 chromosome organization & $3.86 \mathrm{e}-10$ \\
\hline 7 & GO:0006350〜transcription & $7.90 \mathrm{e}-10$ \\
\hline 7 & GO:0006325 chromatin organization & $1.98 \mathrm{e}-09$ \\
\hline 7 & GO:0045449 regulation of transcription & $4.23 e-08$ \\
\hline 7 & GO:0008380 RNA splicing & $1.88 \mathrm{e}-07$ \\
\hline 9 & $\begin{array}{l}\text { hsa04080:Neuroactive ligand-receptor } \\
\text { interaction }\end{array}$ & $1.03 e-04$ \\
\hline 10 & GO:0006955 immune response & $1.61 \mathrm{e}-23$ \\
\hline 10 & hsa05330:Allograft rejection & $7.40 \mathrm{e}-16$ \\
\hline 10 & hsa04940:Type I diabetes mellitus & $5.44 \mathrm{e}-15$ \\
\hline 10 & hsa05332:Graft-versus-host disease & $1.46 \mathrm{e}-12$ \\
\hline 10 & $\begin{array}{l}\text { hsa04672:Intestinal immune network for lgA } \\
\text { production }\end{array}$ & $3.05 e-11$ \\
\hline
\end{tabular}

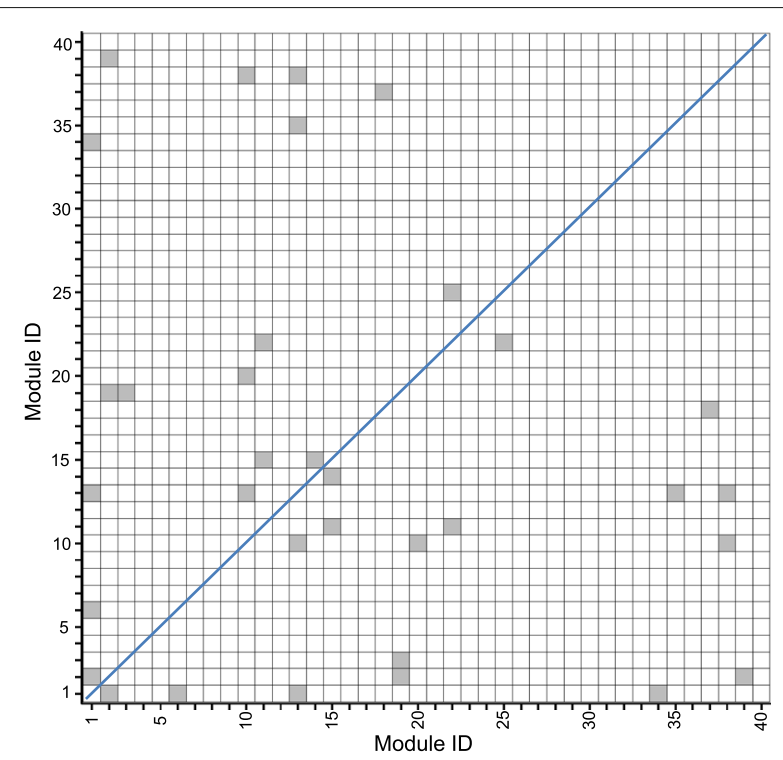

Fig. 6 The overlapping significance level between any two identified modules by SNSVD on the CGP data. Each gray corresponds to a significance overlapping relationship (Hypergeometric test, $p<0.05$ )

than LOSVD by integrating the PPI network. Furthermore, SNSVD obtains a greater number of significant GO BP terms at different levels than LOSVD (one-sided Wilcoxon signed rank test $p$-value $<0.001$ ) (Fig. 7B), showing that incorporating the PPI network does help SNSVD to discover more biological interpretable modules.

\section{Application to the BRCA data sets Data and preprocessing}

We downloaded the processed RNA-seq and miRNA-seq data of Breast invasive carcinoma (BRCA) from TCGA database [30] (Broad GDAC Firehose: http://firebrowse. org/). We firstly filtered out the genes and the miRNAs which are not expressed in more than $70 \%$ samples and the raw gene/miRNA expression values were log2transformed. Secondly, we used the wilcoxon rank sum test to identify differentially expressed genes/miRNAs with bonferroni adjusted $p$-value $<0.05$ between cancer and adjacent normal samples. It causes 9896 differentially expressed genes and 320 differentially expressed miRNAs to be preserved. Thirdly, we imputed the missing values of miRNA and gene expression data by using k-nearest neighbors [31]. Finally, we extracted the matched gene and miRNA expression matrices across cancer and adjacent normal samples, where $\boldsymbol{A}_{1}$ and $\boldsymbol{B}_{1}$ represent gene and miRNA expression data of cancer samples, respectively and (ii) $\boldsymbol{A}_{2}$ and $\boldsymbol{B}_{2}$ represent gene and miRNA expression data of adjacent normal samples, respectively (Fig. 8A). There are 9896 genes and 320 miRNAs, 760 can- 

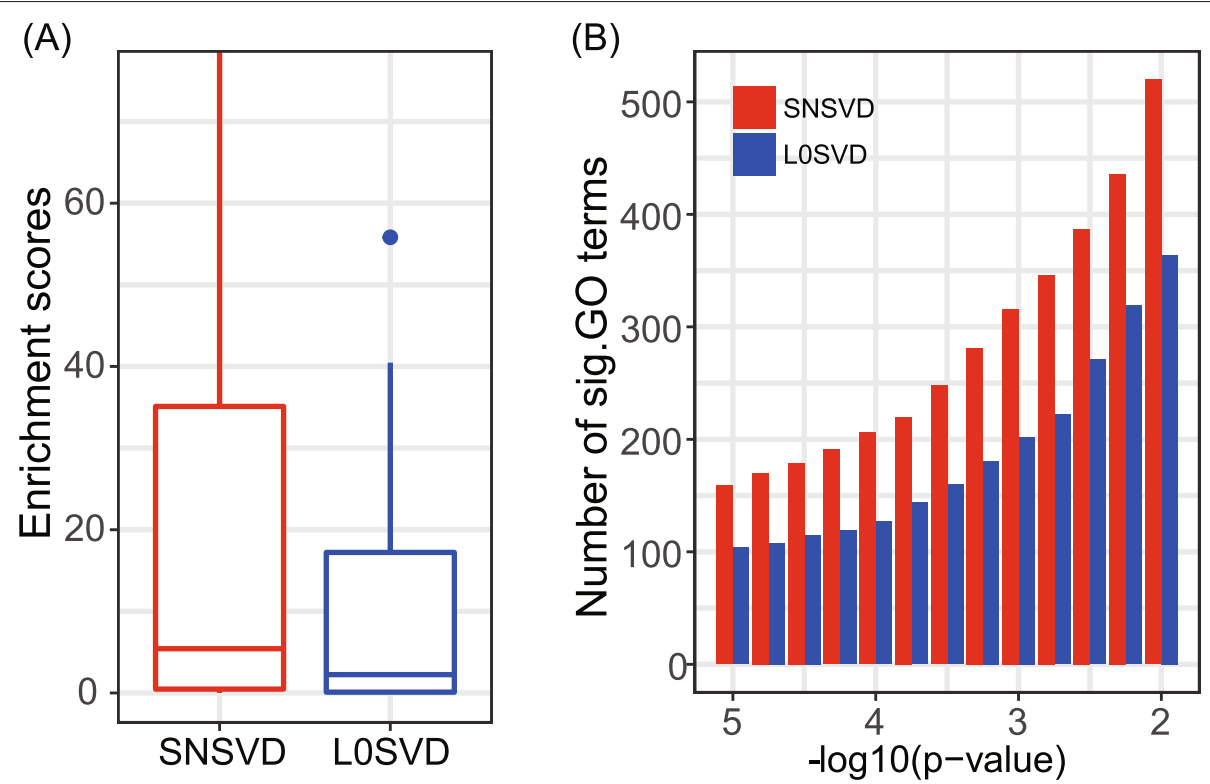

Fig. 7 (A) Comparison of the gene-gene interaction enrichment scores of the identified modules by SNSVD and LOSVD, respectively, on the CGP data. (B) Functional enrichment comparison based on the number of GO BP (Gene Ontology Biological Process) terms

cer samples and 87 adjacent normal samples in the BRCA data sets.

Additionally, we also downloaded a PPI network from Pathway-Commons database [26], and collected a set of cancer genes from the allOnco database (http://www.

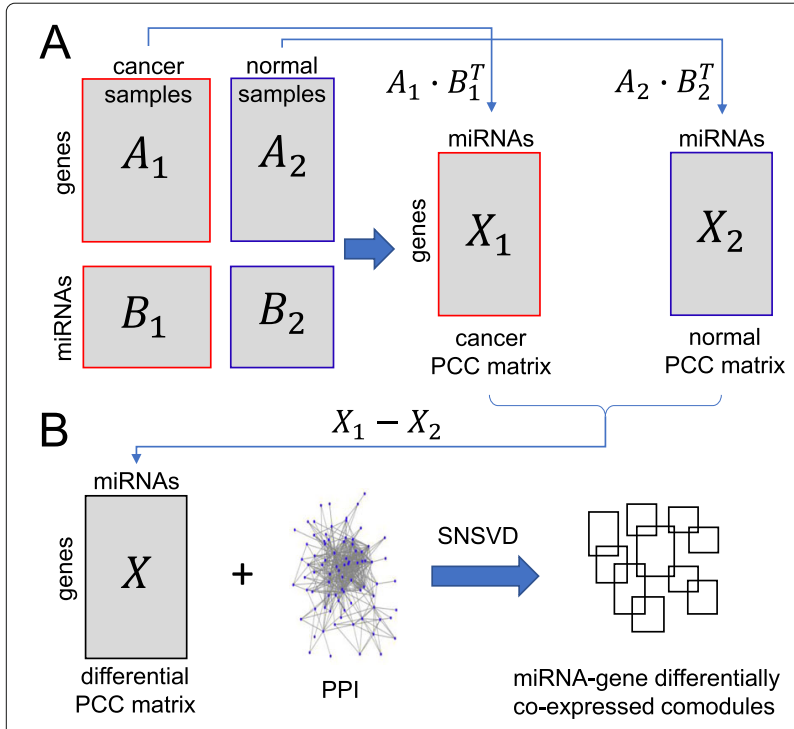

Fig. 8 A framework based on SNSVD method for identifying differentially co-expressed miRNA-gene modules on the BRCA data. A Calculating two miRNA-gene correlation matrices $\boldsymbol{X}_{1}$ and $\boldsymbol{X}_{2}$ using the Pearson correlation method based on the four expression matrices $\boldsymbol{A}_{1}, \boldsymbol{A}_{2}, \boldsymbol{B}_{1}$ and $\boldsymbol{B}_{2}$ whose rows were centered and scaled. B We first obtain the differential correlation matrix $\boldsymbol{X}$ by using $\boldsymbol{X}=\boldsymbol{X}_{1}-\boldsymbol{X}_{2}$. Then, SNSVD is used to identify top ten differentially co-expressed miRNA-gene modules by integrating the differentially co-expressed matrix $\boldsymbol{X}$ and a PPI network bushmanlab.org/links/genelists) which merges some different cancer genes from several databases, and a set of cancer miRNAs from the reference [32].

\section{Identifying differentially co-expressed miRNA-gene modules}

Recent research revealed that some abnormal miRNAgene regulatory relationship plays key roles in tumor progression and development [33-35]. Some computational methods have been proposed for identifying miRNAgene co-expressed modules by using matched miRNA and mRNA expression data of cancer [13, 36-40]. Though power, these methods do not ensure that the miRNAs and genes in a module are differentially expressed between two biological conditions. Besides, some methods have already been developed for differential co-expression analysis [41-44]. However, these methods only focus on single gene expression data analysis. To this end, we proposed a new framework based on SNSVD for analyzing matched miRNA and mRNA expression data between two biological conditions to identify differentially coexpressed miRNA-gene modules (Fig. 8).

Herein, we applied SNSVD to the BRCA data and empirically set $\lambda, k_{v}$ in SNSVD to yield top ten differentially co-expressed modules for each $\sigma$. Each identified miRNA-gene module contain about 10 miRNAs and 100 genes. Formally, a miRNA-gene module contains a miRNA set and a gene set. We found that as $\sigma$ becomes larger, the modules identified by SNSVD contain more edges (Table 2). The results showed SNSVD could overcome the drawbacks of sparse SVD (SNSVD with $\sigma=0$ in Table 2) to capture the modules with more edges by incorporating the PPI network. 
Table 2 Application of SNSVD to the BRCA data. "edge.avg" represents the average of number of edges of modules in the PPI network, and "Fold Change" represents the fold change of "edge.avg" between the identified modules and random modules, and "d.avg" denotes the average of singular values of modules

\begin{tabular}{lllll}
\hline Method & $\boldsymbol{\sigma}$ & edge.avg & FC.avg & d.avg \\
\hline Sparse SVD & 0 & 27.05 & 1.27 & 25.74 \\
SNSVD & 1 & 26.70 & 1.25 & 25.75 \\
SNSVD & 10 & 35.05 & 1.64 & 25.70 \\
SNSVD & 20 & 56.45 & 2.65 & 25.68 \\
SNSVD & 40 & 85.95 & 4.03 & 24.91 \\
SNSVD & 60 & 179.45 & 8.42 & 22.79 \\
SNSVD & 80 & 132.10 & 6.20 & 22.94 \\
SNSVD & 90 & 126.00 & 5.91 & 22.12 \\
SNSVD & 100 & 108.10 & 5.07 & 21.61 \\
SNSVD & 150 & 251.05 & 11.78 & 16.50 \\
SNSVD & 200 & 231.30 & 10.86 & 10.24 \\
\hline
\end{tabular}

Note that SNSVD reduces to a sparse SVD when $\sigma=0$

\section{Functional analysis of modules}

Without loss of generality, the ten modules identified by SNSVD with $\sigma=60$ (See Table 2) were considered for further biological analysis. We found that (i) the average adPCC (absolute differential Pearson Correlation Coefficient) for the identified modules by SNSVD on the BRCA data is larger than the average of all absolute elements of $X$ (Wilcoxon rank-sum test, $p<1 e-16$ ) (Fig. 9A); (ii) more than half of the miRNAs in the $70 \%$ (7 of 10) modules are cancer miRNAs, and $80 \%$ (8 of 10) modules are significantly enriched at least one KEGG/GO BP pathway (Benjamini-Hochberg adjusted $p<0.05$ ); (iii) three modules (module 2, 3 and 8 ) contain significantly more cancer genes with hypergeometric test, $p<0.05$. More results are shown in Fig. 9B. Additionally, we obtained 39 miRNAs and 961 genes by combining the identified ten modules. We found that about 50\% (19 of 39) miRNAs are cancer miRNAs, and about 21\% (203 of 961) genes are cancer genes (hypergeometric test, $p<4.3 e-6$ ).

\section{Discussion}

In our previous work, SSVD has been developed for module discovery and its effectiveness has been demonstrated [13]. However, it cannot integrate the gene network data from PPI network. To this end, we develop the SNSVD method that integrates gene expression data and a gene interaction network to identify underlying gene functional modules. In the SNSVD, we define a sparse network regularized function which is a combination of $L_{1}$-regularized norm and network-regularized norm to make the biclustering process tend to select interacted genes in the prior gene interaction network. Experimental results on the CGP and BRCA data demonstrate that SNSVD can overcome the drawbacks of SSVD. Although SNSVD is an effective method, some further studies are deserved to investigate: (1) extend SNSVD to identify non-linear relationships; (2) extend SNSVD to integrate other omics data, such as DNA methylation data; (3) apply SNSVD to other biological problems.

\section{Conclusions}

In this paper, we presented a Sparse Network-regularized SVD (SNSVD) model for network-based cancer genomics data integration analysis and developed an alternating iterative algorithm to solve the model. By comparing with other representative methods on the simulated data and the real data, we found that SNSVD could find modules with high qualities by integrating the PPI interaction network. By investigating the modules identified by SNSVD on the CGP data, we found that all the genes within the same modules are co-expressed, and most genes in the same modules are connected with each other in the prior PPI network and enriched in at least one gene functional term. Besides, we also applied our method to the BRCA data from TCGA database for identifying ten differentially co-expressed miRNA-gene modules. Some breast cancer related miRNA-gene modules were discovered. To sum up, our work provides a promising way to integrate the network information into the sparse SVD framework, which can help to find biologically significant functional modules and makes the results easily interpreted. An $\mathrm{R}$ package of SNSVD is available at https://github.com/ wenwenmin/SNSVD.

\section{Methods}

Sparse network-regularized SVD (SNSVD) model

Let $X \in \mathbb{R}^{p \times n}$ ( $p$ genes and $n$ samples) be the gene expression data. Suppose $A \in \mathbb{R}^{p \times p}$ is an adjacency matrix of a PPI network, where $A_{i j}=1$ if vertex $i$ and $j$ is connected and $\boldsymbol{A}_{i j}=0$ otherwise. Thus, the normalized Laplacian matrix $\boldsymbol{L}=\left(\boldsymbol{L}_{i j}\right)_{p \times p}$ encoding the PPI network can be defined as:

$$
\boldsymbol{L}_{i j}= \begin{cases}1, & \text { if } i=j \text { and } d_{i} \neq 0 \\ -\frac{A_{i j}}{\sqrt{d_{i} d_{j}}}, & \text { if } i \text { and } j \text { are adjacent } \\ 0, & \text { otherwise }\end{cases}
$$

where $d_{i}=\sum_{j=1}^{p} A_{i j}$. Correspondingly, we have $\boldsymbol{u}^{T} \boldsymbol{L u}=$ $\frac{1}{2} \sum_{i} \sum_{j} A_{i j}\left(\frac{u_{i}}{\sqrt{d_{i}}}-\frac{u_{j}}{\sqrt{d_{j}}}\right)^{2}$, which encourages the estimated coefficients of $\boldsymbol{u}$ to be smooth over adjacent genes in the PPI network $\boldsymbol{A}$ [20]. To further force $\boldsymbol{u}$ to be sparse, we introduce a sparse network-regularized penalty: 


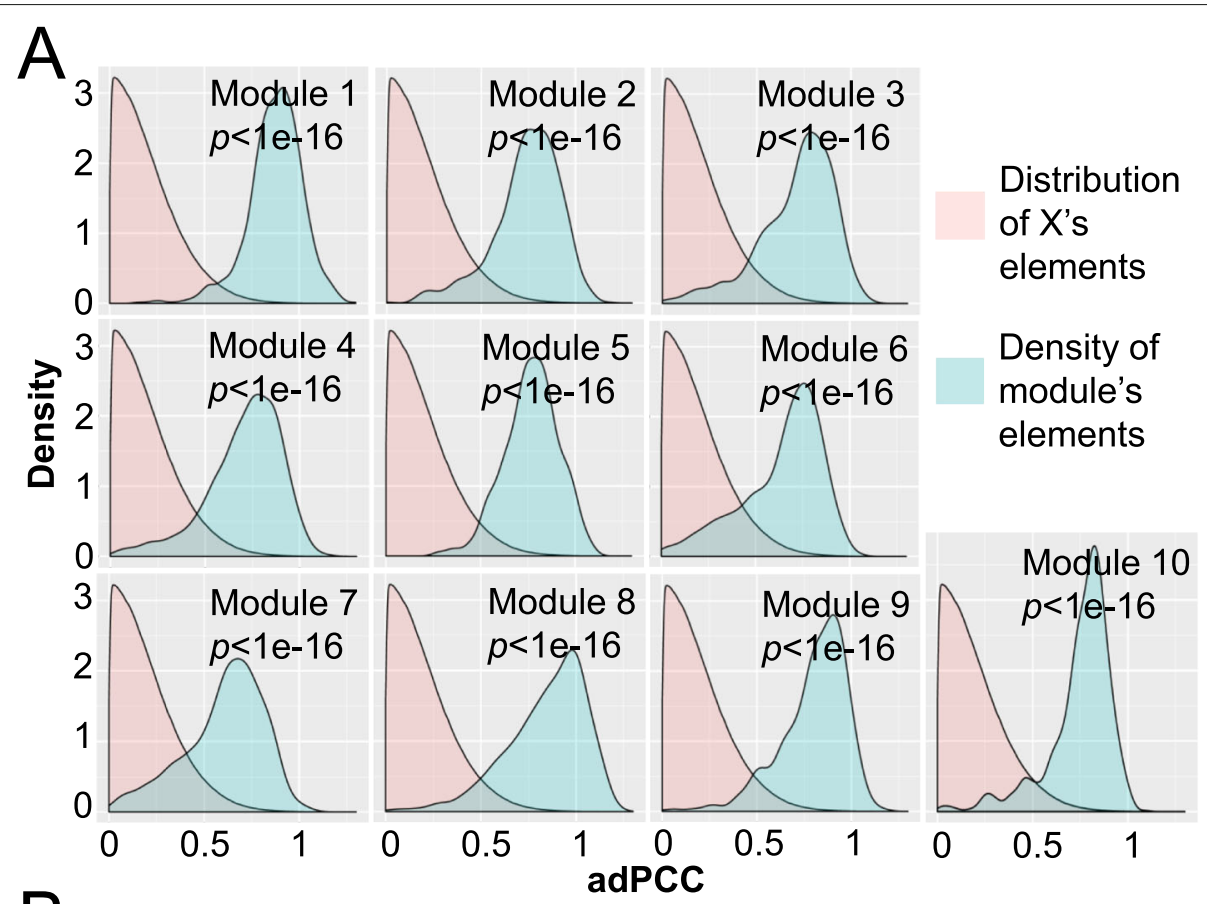

\begin{tabular}{|c|c|c|c|c|c|c|}
\hline Module & $\begin{array}{c}\text { Average } \\
\text { of adPCC }\end{array}$ & $\begin{array}{c}\text { \#Gene } \\
\text { edges }\end{array}$ & $\begin{array}{c}\text { \#Cancer } \\
\text { miRNAs }\end{array}$ & $\begin{array}{c}\text { \#Cancer } \\
\text { genes }\end{array}$ & $\begin{array}{c}\text { \#KEGG } \\
\text { pathways }\end{array}$ & $\begin{array}{c}\text { \#GOBP } \\
\text { pathways }\end{array}$ \\
\hline 1 & 0.874 & 126 & 5 & 23 & 0 & 0 \\
\hline 2 & 0.742 & 636 & 5 & 32 & 11 & 131 \\
\hline 3 & 0.712 & 413 & 4 & 26 & 7 & 134 \\
\hline 4 & 0.707 & 73 & 5 & 20 & 0 & 3 \\
\hline 5 & 0.769 & 85 & 6 & 16 & 0 & 2 \\
\hline 6 & 0.640 & 709 & 4 & 12 & 14 & 128 \\
\hline 7 & 0.604 & 763 & 3 & 21 & 4 & 170 \\
\hline 8 & 0.843 & 229 & 7 & 26 & 11 & 47 \\
\hline 9 & 0.802 & 136 & 7 & 20 & 2 & 62 \\
\hline 10 & 0.748 & 89 & 6 & 20 & 0 & 0 \\
\hline
\end{tabular}

Fig. 9 Biological function analysis of the identified ten differentially co-expressed miRNA-gene modules by SNSVD with $\sigma=60$. A For each module, the distribution (pink area) is fitted based on the absolute values of all the elements in the differentially co-expressed matrix $\boldsymbol{X}$, and the distribution (light blue area) is fitted based on the absolute values of the elements from the module corresponding submatrix in $\boldsymbol{X}$. $P$-values were computed by using permutation test. B For each module, "Average of adPCC" is the average of the absolute values of the elements from the module

corresponding submatrix in $\boldsymbol{X}$. "\#Gene edges", "\#Cancer gene", "\#Cancer miRNA", "\#KEGG pathways" and "\#GOBP pathways" represent the number of interaction edges, cancer genes, cancer miRNAs, significantly enriched KEGG pathways and GO BP pathways (Benjamini-Hochberg adjusted $p<0.05)$, respectively

$$
P_{1}(\boldsymbol{u})=\lambda\|\boldsymbol{u}\|_{1}+\sigma \boldsymbol{u}^{T} \boldsymbol{L u},
$$

where $\lambda$ and $\sigma$ are two parameters. In the penalty (3), the $L_{1}$ norm $\left(\|\boldsymbol{u}\|_{1}\right)$ is to induce sparsity in $\boldsymbol{u}$; and the quadratic Laplacian norm $\left(\boldsymbol{u}^{T} \boldsymbol{L} \boldsymbol{u}\right)$ makes the selected genes tend to connect with each other in the PPI network.

To integrate the network information in SVD framework, we present a sparse network-regularized SVD (SNSVD) model as follows:

$$
\begin{array}{cl}
\underset{\boldsymbol{u}, \boldsymbol{v}, d}{\operatorname{minimize}} & \left\|\boldsymbol{X}-d \boldsymbol{u} \boldsymbol{v}^{T}\right\|_{F}^{2} \\
\text { subject to } & \|\boldsymbol{u}\|_{2}^{2} \leq 1, \lambda\|\boldsymbol{u}\|_{1}+\sigma \boldsymbol{u}^{T} \boldsymbol{L} \boldsymbol{u} \leq c_{1}, \\
& \|\boldsymbol{v}\|_{2}^{2} \leq 1,\|\boldsymbol{v}\|_{0} \leq k_{v}
\end{array}
$$

where $c_{1}$ and $k_{v}$ are two parameters to control the number of selected genes and samples separately. As for the samples, we simply use a $L_{0}$-regularized penalty on $\boldsymbol{v}$ sample variables (corresponding to sample variables) to induce 
sparseness. Compared to $L_{1}$-norm, $L_{0}$-norm is known as the most essential sparsity measure and has nice theoretical properties $[15,45]$.

\section{SNSVD algorithm}

Since $\left\|\boldsymbol{X}-d \boldsymbol{u} \boldsymbol{v}^{T}\right\|_{F}^{2}=\operatorname{tr}\left(\boldsymbol{X} \boldsymbol{X}^{T}\right)+d^{2} \operatorname{tr}\left(\boldsymbol{u} \boldsymbol{v}^{T} \boldsymbol{v} \boldsymbol{u}^{T}\right)-$ $2 d \boldsymbol{u}^{T} \boldsymbol{X} \boldsymbol{v}$, where $\operatorname{tr}(\cdot)$ denotes the trace of a matrix; Both $\boldsymbol{u}$ and $\boldsymbol{v}$ are guaranteed to be two unit vectors, $\operatorname{tr}\left(\boldsymbol{u} \boldsymbol{v}^{T} \boldsymbol{v} \boldsymbol{u}^{T}\right)=\operatorname{tr}\left(\boldsymbol{u}^{T} \boldsymbol{u} \boldsymbol{v}^{T} \boldsymbol{v}\right)=1$. Minimizing $\| \boldsymbol{X}-$ $d \boldsymbol{u} \boldsymbol{v}^{T} \|_{F}^{2}$ in Eq. (4) is equivalent to minimizing $-\boldsymbol{u}^{T} \boldsymbol{X} \boldsymbol{v}$. Although there are three parameters $\boldsymbol{u}, \boldsymbol{v}$ and $d$ to be optimized in Eq. (4). It is notable that once $\boldsymbol{u}$ and $\boldsymbol{v}$ are fixed, then $d$ can be determined $d=\boldsymbol{u}^{T} \boldsymbol{X} \boldsymbol{v}$ in Eq. (4). Thus, to solve Eq. (4), we just need to optimize $\boldsymbol{u}$ and $\boldsymbol{v}$. Inspired by Ref. [46], we present an alternating iterative strategy to solve $\boldsymbol{u}$ and $\boldsymbol{v}$, i.e., fixing $\boldsymbol{v}$ to update $\boldsymbol{u}$ and fixing $\boldsymbol{u}$ to update $\boldsymbol{v}$.

Fixing $v$ in Eq. (4), it is equivalent to solve the following sub-problem:

$$
\begin{array}{ll}
\underset{\boldsymbol{u}}{\operatorname{minimize}} & -\boldsymbol{u}^{T} \boldsymbol{X} \boldsymbol{v} \\
\text { subject to } & \|\boldsymbol{u}\|_{2}^{2} \leq 1, \lambda\|\boldsymbol{u}\|_{1}+\sigma \boldsymbol{u}^{T} \boldsymbol{L} \boldsymbol{u} \leq c_{1} .
\end{array}
$$

Let $z=X \boldsymbol{v}$, the optimization problem in (5) can be redefined as follows:

$$
\begin{array}{ll}
\underset{\boldsymbol{u}}{\operatorname{minimize}} & -\boldsymbol{u}^{T} \boldsymbol{z} \\
\text { subject to } & \|\boldsymbol{u}\|_{2}^{2} \leq 1, \lambda\|\boldsymbol{u}\|_{1}+\sigma \boldsymbol{u}^{T} \boldsymbol{L} \boldsymbol{u} \leq c_{1} .
\end{array}
$$

To solve it, we write its Lagrangian form as follows:

$$
\mathcal{L}(\boldsymbol{u})=-\boldsymbol{u}^{T} \boldsymbol{z}+\eta \boldsymbol{u}^{T} \boldsymbol{u}+\lambda\|\boldsymbol{u}\|_{1}+\sigma \boldsymbol{u}^{T} \boldsymbol{L} \boldsymbol{u},
$$

where $\lambda \geq 0, \eta \geq 0, \sigma \geq 0$. In order to facilitate the calculation without loss of generality, we use $\frac{1}{2} \eta$ instead of $\eta, \frac{1}{2} \sigma$ instead of $\sigma$, then Eq. (7) can be rewritten as:

$$
\mathcal{L}(\boldsymbol{u})=-\boldsymbol{u}^{T} \boldsymbol{z}+\frac{1}{2} \eta \boldsymbol{u}^{T} \boldsymbol{u}+\lambda\|\boldsymbol{u}\|_{1}+\frac{1}{2} \sigma \boldsymbol{u}^{T} \boldsymbol{L} \boldsymbol{u} .
$$

It is a convex function with respect to $\boldsymbol{u}$, therefore its optimal solution can be characterized by some subgradient equations (see e.g., [47]). Since $\boldsymbol{L}=\boldsymbol{I}-$ $\boldsymbol{D}^{-1 / 2} \boldsymbol{A} \boldsymbol{D}^{-1 / 2}$ (based on Eq. 2). For convenience, let $\boldsymbol{W}=$ $\boldsymbol{I}-\boldsymbol{L}=\boldsymbol{D}^{-1 / 2} \boldsymbol{A} \boldsymbol{D}^{-1 / 2}(\boldsymbol{D}$ is a diagonal matrix and $\left.D_{i i}=\sum_{j} A_{i j}\right)$, then we have the sub-gradient equations of Eq. (8) as:

$$
\frac{\partial \mathcal{L}}{\partial \boldsymbol{u}_{j}}=-\boldsymbol{z}_{j}+\eta \boldsymbol{u}_{j}+\lambda s_{j}+\sigma \boldsymbol{u}_{j}-\sigma \boldsymbol{W}_{j} \boldsymbol{u}=0, j=1, \cdots, p .
$$

where $s_{j}=\operatorname{sign}\left(\boldsymbol{u}_{j}\right)$ if $\boldsymbol{u}_{j} \neq 0$ and $s_{j} \in\{t,|t| \leq 1\}$ otherwise; and $W_{j}$ is the $j$ th row of matrix $W$. Let the solution of (8) be $\widehat{\boldsymbol{u}}=\left(\widehat{\boldsymbol{u}}_{1}, \widehat{\boldsymbol{u}}_{2}, \cdots, \widehat{\boldsymbol{u}}_{p}\right)$. By using a coordinate descent method $[48,49]$, we obtain the following coordinate update rule for $\widehat{\boldsymbol{u}}_{j}$ :

$$
\widehat{\boldsymbol{u}}_{j}= \begin{cases}0 & \text { if }\left|\boldsymbol{z}_{j}+\sigma \boldsymbol{W}_{j} \widehat{\boldsymbol{u}}\right| \leq \lambda, \\ \frac{\boldsymbol{z}_{j}+\sigma \boldsymbol{W}_{j} \widehat{\boldsymbol{u}}-\lambda \operatorname{sign}\left(\widehat{\boldsymbol{u}}_{j}\right)}{\eta+\sigma} & \text { otherwise. }\end{cases}
$$

Define $\mathcal{S}(a, \lambda)=\operatorname{sign}(a)(|a|-\lambda)_{+}$, we have $\widehat{\boldsymbol{u}}_{j}=$ $\mathcal{S}\left(\boldsymbol{z}_{j}+\sigma \boldsymbol{W}_{j} \widehat{\boldsymbol{u}}, \lambda\right) /(\eta+\sigma)$. Let $\breve{\boldsymbol{u}}_{j}=\left(\boldsymbol{z}_{j}+\sigma \boldsymbol{W}_{j} \widehat{\boldsymbol{u}}, \lambda\right)$ and $\breve{\boldsymbol{u}}=\left(\breve{\boldsymbol{u}}_{1}, \ldots, \breve{\boldsymbol{u}}_{p}\right)^{T}$, we can obtain a normalized solution $\boldsymbol{u}=\frac{\widehat{\boldsymbol{u}}}{\|\widehat{\boldsymbol{u}}\|_{2}}=\frac{\breve{\boldsymbol{u}}}{\|\breve{\boldsymbol{u}}\|_{2}}$. In a word, we use a coordinate descent method to minimize Eq. (8) and update one $\boldsymbol{u}_{j}$ at a time while keeping $\boldsymbol{u}_{k}$ fixed for all $k \neq j$.

Fixing $\boldsymbol{u}$ in Eq. (4), it is equivalent to solve the following sub-problem:

$$
\begin{array}{ll}
\underset{\boldsymbol{v}}{\operatorname{minimize}} & \left\|\boldsymbol{X}-d \boldsymbol{u} \boldsymbol{v}^{T}\right\|_{F}^{2} \\
\text { subject to } & \|\boldsymbol{v}\|_{2}^{2} \leq 1,\|\boldsymbol{v}\|_{0} \leq k_{\boldsymbol{v}} .
\end{array}
$$

Let $\boldsymbol{z}_{v}=\boldsymbol{X}^{T} \boldsymbol{u}, \widehat{\boldsymbol{v}}=d \boldsymbol{v}$, we thus have $\left\|\boldsymbol{X}-d \boldsymbol{u} \boldsymbol{v}^{T}\right\|_{F}^{2}=$ $\left\|\boldsymbol{z}_{v}-\widehat{\boldsymbol{v}}\right\|_{2}^{2}+c$, where $c=\operatorname{tr}\left(\boldsymbol{X}^{T} \boldsymbol{X}\right)-\boldsymbol{u}^{T} \boldsymbol{X} \boldsymbol{X}^{T} \boldsymbol{u}$. Obviously $c$ is a constant value with respect to $v$. Thus problem (11) is equivalent to:

$$
\min _{\widehat{\boldsymbol{v}}}\left\|\boldsymbol{z}_{v}-\widehat{\boldsymbol{v}}\right\|_{2}^{2} \text {, subject to }\|\widehat{\boldsymbol{v}}\|_{0} \leq k_{v} \text {. }
$$

Its optimal solution is $\widehat{\boldsymbol{v}}=\boldsymbol{z}_{v} \bullet I\left(\left|\boldsymbol{z}_{v}\right| \geq\left|\boldsymbol{z}_{v}\right|_{\left(k_{v}\right)}\right)$ where $I(\cdot)$ is the indicator function and " $\bullet "$ is point multiplication function, and $\left|z_{v}\right|_{(i)}$ denotes the $i$-th order statistic of $\left|\boldsymbol{z}_{v}\right|$, i.e. $\left|\boldsymbol{z}_{v}\right|_{(1)} \geq\left|\boldsymbol{z}_{v}\right|_{(2)} \geq, \ldots, \geq\left|\boldsymbol{z}_{v}\right|_{(n)}$. In other words, we only keep the $k_{v}$ variables of $\boldsymbol{z}_{v}$ corresponding to its $k_{v}$ largest absolute values. The normalized optimal solution of Eq. (11) is $\boldsymbol{v}=\widehat{\boldsymbol{v}} /\|\widehat{\boldsymbol{v}}\|_{2}$, i.e.,

$$
\boldsymbol{v}=\frac{\boldsymbol{z}_{v} \bullet I\left(\left|\boldsymbol{z}_{v}\right| \geq\left|\boldsymbol{z}_{v}\right|_{\left(k_{v}\right)}\right)}{\left\|\boldsymbol{z}_{v} \bullet I\left(\left|\boldsymbol{z}_{v}\right| \geq\left|\boldsymbol{z}_{v}\right|_{\left(k_{v}\right)}\right)\right\|_{2}} .
$$

Finally, we develop an alternating iterative algorithm by alternately updating $\boldsymbol{u}$ and $\boldsymbol{v}$ to solve SNSVD model. The details of this algorithm is given in Algorithm 1, and its time complexity is $\mathcal{O}\left(\operatorname{Tn} p+T p^{2}+T n^{2}\right)$, where $T$ is the number of iterations.

\section{Convergence analysis of SNSVD algorithm}

Next, we give the convergence analysis of Algorithm 1. In fact, Algorithm 1 is to solve the Lagrangian form of problem (4) as follows:

$$
\begin{array}{cl}
\underset{\boldsymbol{u}, \boldsymbol{v}, d}{\operatorname{minimize}} & -\boldsymbol{u}^{T} \boldsymbol{X} \boldsymbol{v}+\lambda\|\boldsymbol{u}\|_{1}+\sigma \boldsymbol{u}^{T} \boldsymbol{L} \boldsymbol{u} \\
\text { subject to } & \|\boldsymbol{u}\|_{2}^{2} \leq 1,\|\boldsymbol{v}\|_{2}^{2} \leq 1,\|\boldsymbol{v}\|_{0} \leq k_{v} .
\end{array}
$$

Let $H(\boldsymbol{u}, \boldsymbol{v})=-\boldsymbol{u}^{T} \boldsymbol{X} \boldsymbol{v}+\sigma \boldsymbol{u}^{T} \boldsymbol{L u}, f(\boldsymbol{u})=\rho(\boldsymbol{u})+\lambda\|\boldsymbol{u}\|_{1}$ and $g(\boldsymbol{v})=\rho(\boldsymbol{v})+\tau\left(\boldsymbol{v}, k_{v}\right)$ where

$$
\begin{gathered}
\rho(\boldsymbol{u})= \begin{cases}0, & \text { if }\|\boldsymbol{u}\|_{2}^{2} \leq 1 \\
+\infty, & \text { otherwise. }\end{cases} \\
\rho(\boldsymbol{v})= \begin{cases}0, & \text { if }\|\boldsymbol{v}\|_{2}^{2} \leq 1 \\
+\infty, & \text { otherwise. }\end{cases} \\
\tau\left(\boldsymbol{v}, k_{v}\right)= \begin{cases}0, & \text { if }\|\boldsymbol{v}\|_{0} \leq k_{v} \\
+\infty, & \text { otherwise. }\end{cases}
\end{gathered}
$$




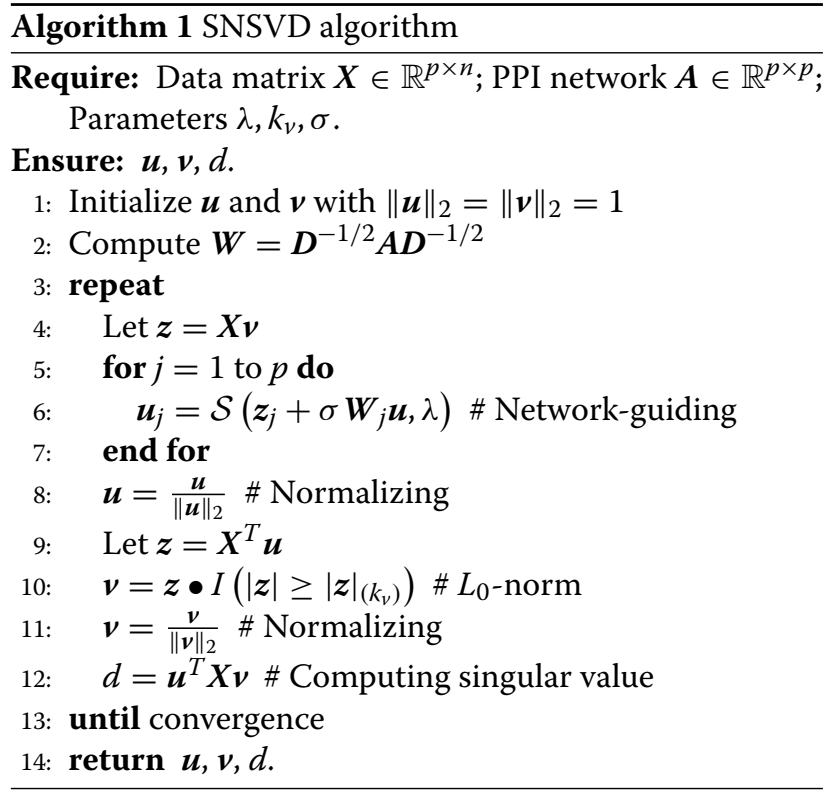

Therefore the Lagrangian form of problem (4) can be written as $F(\boldsymbol{u}, \boldsymbol{v})=H(\boldsymbol{u}, \boldsymbol{v})+f(\boldsymbol{u})+g(\boldsymbol{v})$ which is a semialgebraic function [46]. Based on the Theorem 1 in [46], Algorithm 1 converges to a critical point of $F(\boldsymbol{u}, \boldsymbol{v})$.

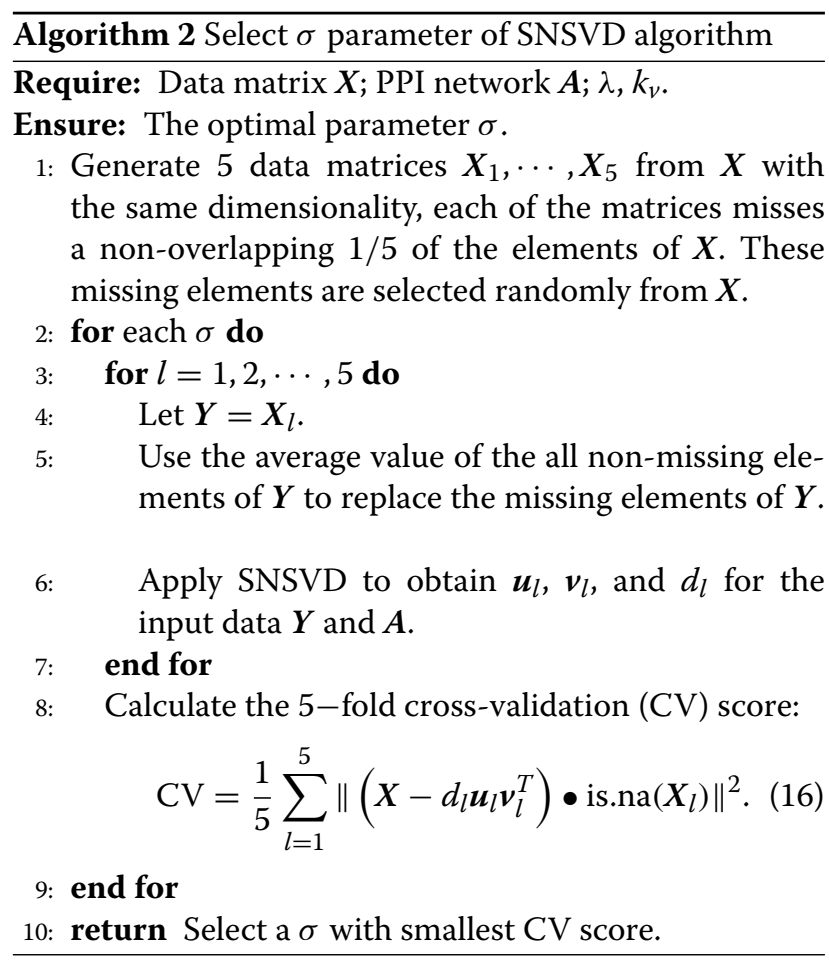

\section{Parameter selection of SNSVD algorithm}

As to $\lambda$ and $k_{v}$ 's choice in Algorithm 1 when it is applied to the CGP gene expression data, we select a suitable $\lambda$ to force the estimated $\boldsymbol{u}$ only containing 200 nonzero elements which is beneficial for further analysis of the biological function of the module and set $k_{v}=50$ (control the sample sparsity) which ensures that the number of samples within in the module is approximately the same as the number of samples of a subtype. As to $\sigma$ 's choice in Algorithm 1, we present a 5-fold cross-validation framework (Algorithm 2). To this end, we define a binary matrix is.na $(X)$ with the same size of $\boldsymbol{X}$ and the elements are 1 if they are missing in $X, 0$ otherwise.

\section{Learning multiple pairs of singular vectors using SNSVD}

It is notable that every run of Algorithm 1 can only obtain a pair of sparse singular vectors $\boldsymbol{u}$ and $\boldsymbol{v}$ (Fig. 1). In order to identify multiple modules, we can repeat running Algorithm 1. After each turn of the iteration, we use the obtained $\boldsymbol{u}, \boldsymbol{v}$ and $d$ to modify the gene expression data $X,\left(X:=X-d u v^{T}\right)$, the modified $X$ is then used as the new input data for the next run to obtain the next pair of singular vectors. Moreover, we notice that Algorithm 1 may get different local optima with different initials, we run Algorithm 1 five times with different initials which are generated according to the multivariate standard normal distribution and choose the best one as the final solution of each turn. The detailed procedure is described in Algorithm 3.

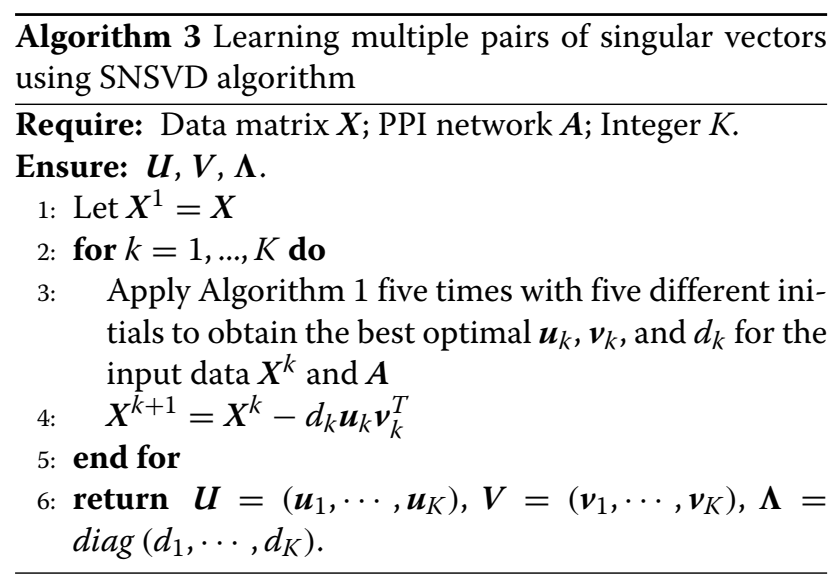

\section{Modularity score}

To assess whether the genes within the same module are co-expressed/correlated, we use a modularity score to describe the overall co-expression of genes within the module. For a given module $k$ containing $p_{k}$ genes and $n_{k}$ samples, we first calculate the correlation between gene $i$ and $j$ across the $n_{k}$ samples, denoted as $w_{i j}$. For convenience, we force to set $w_{i i}=0$ for each $i$. Then the modularity score of the module can be defined as: 


$$
\text { Modularity }=\frac{1}{p_{k} \cdot\left(p_{k}-1\right)} \sum_{i=1}^{p_{k}} \sum_{j=1}^{p_{k}}\left|w_{i j}\right| .
$$

Intuitively, if a module has a high modularity score, then the genes within the module is highly co-expressed.

\section{Gene-gene interaction enrichment score}

In order to evaluate whether the genes within the same module are tightly connected in the prior PPI network, we use the right tailed hypergeometric test to compute a significance level of each module. Suppose that the PPI network contains $n$ genes and $m$ edges, and module $i$ contains $n_{i}$ genes and $m_{i}$ edges, then the significance level of module $k$ can be calculated via the following equation:

$$
p(i)=\sum_{x<m_{i}} \frac{\left(\begin{array}{l}
m \\
x
\end{array}\right)\left(\begin{array}{l}
N-m \\
m_{i}-x
\end{array}\right)}{\left(\begin{array}{l}
N \\
N_{i}
\end{array}\right)},
$$

where $N=\left(\begin{array}{c}n \\ 2\end{array}\right)$ and $N_{i}=\left(\begin{array}{c}n_{i} \\ 2\end{array}\right)$. Accordingly, we can define the gene-gene interaction enrichment score $s(i)$ of the module $i$ by the following formula:

$$
s(i)=-\log _{10}(p(i))
$$

The higher the gene-gene interaction enrichment score is, the denser the genes connect with each other. If the score is higher than 1.3, then the genes are significantly inter-connected with each other in the PPI network.

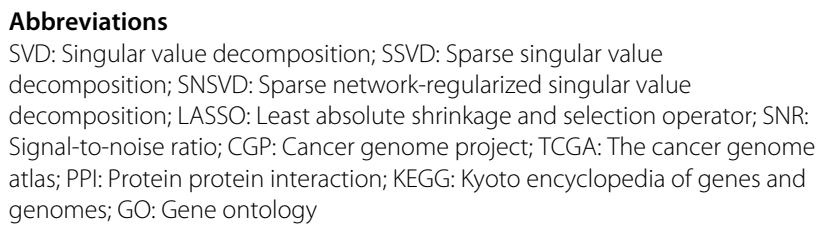

\section{Acknowledgements}

Wenwen Min would like to thank the support of National Center for Mathematics and Interdisciplinary Sciences, Academy of Mathematics and Systems Science, CAS during his visit.

\section{About this supplement \\ This article has been published as part of BMC Genomic Data Volume 22 Supplement 1, 2021: Proceedings of the 2019 International Conference on Intelligent Computing (ICIC 2019): genomic data. The full contents of the supplement are available online at https://bmcgenomdata.biomedcentral. com/articles/supplements/volume-22-supplement-1.}

\section{Authors' contributions}

The authors wish it to be known that, in their opinion, F.Z. and W.M. should be regarded as Joint First Authors. F.Z., W.M., J.Liu and J.Li developed the methodology and executed experiments. F.Z. and W.M. drafted the manuscript, J.Liu and J.Li revised the manuscript. W.M., J.Liu and J.Li supervised this study. The authors read and approved the final manuscript.

\section{Funding}

This work was supported by the National Science Foundation of China [No. 61802157] and Natural Science Foundation of Jiangxi Province of China [No. 20192BAB217004]. The funding bodies had no role in the design of the study or collection, analysis and interpretation of the data.

\section{Availability of data and materials}

The CGP datasets used during the current study are available in the Cancer Genome Project (CGP) repository (http://www.cancerrxgene.org/downloads). And the BRCA datasets used during the current study are available in the TCGA repository (Broad GDAC Firehose: http://firebrowse.org/).

\section{Declarations}

Ethics approval and consent to participate Not applicable.

\section{Consent for publication}

Not applicable.

\section{Competing interests}

The authors declare that they have no competing interests.

\section{Author details}

'State Key Laboratory of Nuclear Resources and Environment and School of Water Resources and Environmental Engineering, East China University of Technology, 330013 Nanchang, China. ${ }^{2}$ State Key Laboratory of Nuclear Resources and Environment and School of Chemistry, Biology and Materials Science, East China University of Technology, 330013 Nanchang, China. ${ }^{3}$ School of Computer Science, Wuhan University, 430072 Wuhan, China. ${ }^{4}$ School of Mathematics and Computer Science, Jiangxi Science and Technology Normal University, 330038 Nanchang, China. ${ }^{5}$ Information School, Yunnan University, 650091 Kunming, China.

Received: 26 September 2021 Accepted: 20 October 2021

Published: 10 December 2021

\section{References}

1. Wan Q, Dingerdissen H, Fan Y, Gulzar N, Pan Y, Wu TJ, Yan C, Zhang H, Mazumder R. Bioxpress: An integrated RNA-seq-derived gene expression database for pan-cancer analysis. Database (Oxford). 2015;2015:1-13.

2. Vallejos CA, Risso D, Scialdone A, Dudoit S, Marioni JC. Normalizing single-cell RNA sequencing data: challenges and opportunities. Nat Methods. 2017;14(6):565-71.

3. Iorio F, Knijnenburg TA, Vis DJ, Bignell GR, Menden MP, Schubert M, Aben N, Gonçalves E, Barthorpe S, Lightfoot H, et al. A landscape of pharmacogenomic interactions in cancer. Cell. 2016;166(3):740-54.

4. Lee M, Shen H, Huang JZ, Marron J. Biclustering via sparse singular value decomposition. Biometrics. 2010;66(4):1087-95.

5. Liquet B, de Micheaux PL, Hejblum BP, Thiébaut R. Group and sparse group partial least square approaches applied in genomics context. Bioinformatics. 2015;32(1):35-42.

6. Min W, Liu J, Zhang S. Edge-group sparse pca for network-guided high dimensional data analysis. Bioinformatics. 2018;34(20):3479-87.

7. Liu X, Chang X, Liu R, Yu X, Chen L, Aihara K. Quantifying critical states of complex diseases using single-sample dynamic network biomarkers. PLoS Comput Biol. 2017;13(7):1005633.

8. YuX, Zhang J, Sun S, Zhou X, Zeng T, Chen L. Individual-specific edge-network analysis for disease prediction. Nucleic Acids Res. 2017;45(20):170.

9. Eren K, Deveci M, Küçüktunç O, Ümit V. Çatalyürek: A comparative analysis of biclustering algorithms for gene expression data. Brief Bioinforma. 2013;14(3):279-92.

10. Sill M, Kaiser S, Benner A, Kopp-Schneider A. Robust biclustering by sparse singular value decomposition incorporating stability selection. Bioinformatics. 2011;27(15):2089-97.

11. Oghabian A, Kilpinen S, Hautaniemi S, Czeizler E. Biclustering methods: Biological relevance and application in gene expression analysis. PLoS ONE. 2014;9(3):.

12. Chen S, Liu J, Zeng T. Measuring the quality of linear patterns in biclusters. Methods. 2015;83:18-27.

13. Min W, Liu J, Luo F, Zhang S. A two-stage method to identify joint modules from matched microRNA and mRNA expression data. IEEE Trans Nanobiosci. 2016;15(4):362-370.

14. Yang D, Ma Z, Buja A. Rate optimal denoising of simultaneously sparse and low rank matrices. J Mach Learn Res. 2016;17(1):3163-89. 
15. Asteris M, Kyrillidis A, Koyejo O, Poldrack R. A simple and provable algorithm for sparse diagonal CCA. In: International Conference on Machine Learning; 2016. p. 1148-1157.

16. Sokolov A, Carlin DE, Paull EO, Baertsch R, Stuart JM. Pathway-based genomics prediction using generalized elastic net. PLoS Comput Biol. 2016;12(3):e1004790.

17. Hill SM, Heiser LM, et al. Inferring causal molecular networks: empirical assessment through a community-based effort. Nat Methods. 2016;13(4): 310-8.

18. Enrico G. Using prior knowledge from cellular pathways and molecular networks for diagnostic specimen classification. Brief Bioinforma. 2016;17(3):440-52.

19. Lee $\mathrm{E}$, Chuang H-Y, Kim J-W, Ideker T, Lee D. Inferring pathway activity toward precise disease classification. PLoS Comput Biol. 2008;4(11): e1000217.

20. Li C, Li H. Network-constrained regularization and variable selection for analysis of genomic data. Bioinformatics. 2008;24(9):1175-82.

21. Sun H, Feng R, Lin W, Li H. Network-regularized high-dimensional cox regression for analysis of genomic data. Stat Sin. 2013;24(3):1433-59.

22. Chen J, Zhang S. Integrative analysis for identifying joint modular patterns of gene-expression and drug-response data. Bioinformatics. 2016;32(11):1724-32.

23. Zhu F, Liu J, Min W. Gene functional module discovery via integrating gene expression and ppi network data. In: International Conference on Intelligent Computing; 2019. p. 116-126. https://doi.org/10.1007/978-3030-26969-2_11.

24. Tibshirani R. Regression shrinkage and selection via the lasso. J R Stat Soc Ser B (Methodological). 1996;58(1):267-88.

25. Fan J, Li R. Variable selection via nonconcave penalized likelihood and its oracle properties. J Am Stat Assoc. 2001;96(456):1348-60.

26. Cerami EG, Gross BE, et al. Pathway commons, a web resource for biological pathway data. Nucleic Acids Res. 2011;39(Database Issue): 685-90.

27. Huang DW, Sherman BT, Lempicki RA. Systematic and integrative analysis of large gene lists using DAVID bioinformatics resources. Nat Protocol. 2009;4(1):44-57.

28. Leeksma OC, de Miranda NF, Veelken H. Germline mutations predisposing to diffuse large B-cell lymphoma. Blood Cancer J. 2017;7(2): 532.

29. Disis ML. Immune regulation of cancer. J Clin Oncol. 2010;28(29):4531-8.

30. Lander ES, Park PJ. The cancer genome atlas pan-cancer analysis project. Nat Genet. 2013;45(10):1113-20.

31. Troyanskaya $\mathrm{O}$, Cantor $\mathrm{M}$, et al. Missing value estimation methods for DNA microarrays. Bioinformatics. 2001;17(6):520-5.

32. Xie $B$, Ding $Q$, Han $H$, Wu D. miRCancer: a microRNA cancer association database constructed by text mining on literature. Bioinformatics. 2013;29(5):638-44.

33. Garzon R, Calin GA, Croce CM. MicroRNAs in cancer. Ann Rev Med. 2009;60:167-79.

34. Adams BD, Kasinski AL, Slack FJ. Aberrant regulation and function of microRNAs in cancer. Curr Biol. 2014;24(16):762-76.

35. Iorio MV, Croce CM. MicroRNAs in cancer: small molecules with a huge impact. J Clin Oncol. 2009;27(34):5848.

36. Zhang S, Li Q, Liu J, Zhou XJ. A novel computational framework for simultaneous integration of multiple types of genomic data to identify microRNA-gene regulatory modules. Bioinformatics. 2011;27(13):401-9.

37. Zhang S, Liu C-C, Li W, Shen H, Laird PW, Zhou XJ. Discovery of multi-dimensional modules by integrative analysis of cancer genomic data. Nucleic Acids Res. 2012;40(19):9379-91.

38. Bryan $\mathrm{K}$, et al. Discovery and visualization of miRNA-mRNA functional modules within integrated data using bicluster analysis. Nucleic Acids Res. 2013;42(3):17.

39. Li Y, Liang C, Wong K-C, Luo J, Zhang Z. Mirsynergy: detecting synergistic miRNA regulatory modules by overlapping neighbourhood expansion. Bioinformatics. 2014;30(18):2627-35.

40. Jin D, Lee H. A computational approach to identifying gene-microRNA modules in cancer. PLoS Comput Biol. 2015;11(1):1004042.

41. Tesson BM, Breitling R, Jansen RC. DiffCoEx: a simple and sensitive method to find differentially coexpressed gene modules. BMC Bioinformatics. 2010;11:497.

42. Ideker T, Krogan NJ. Differential network biology. Mol Syst Biol. 2012;8:565
43. Ha MJ, Baladandayuthapani V, Do K-A. Dingo: differential network analysis in genomics. Bioinformatics. 2015;31(21):3413-20.

44. Zhu L, et al. MetaDCN: meta-analysis framework for differential co-expression network detection with an application in breast cancer. Bioinformatics. 2016;33(8):1121-9.

45. Yang F, Shen Y, Liu ZS. The proximal alternating iterative hard thresholding method for $L_{0}$ minimization, with complexity $\mathcal{O}(1 / \sqrt{k})$. J Comput Appl Math. 2017;311:115-29.

46. Bolte J, Sabach S, Teboulle M. Proximal alternating linearized minimization for nonconvex and nonsmooth problems. Math Program. 2014;146(1-2):459-94

47. Nesterov Y. Primal-dual subgradient methods for convex problems. Math Program. 2009;120(1):221-259.

48. Friedman J, Hastie T, Höfling H, Tibshirani R, et al. Pathwise coordinate optimization. Ann Appl Stat. 2007;1(2):302-332.

49. Friedman J, Hastie T, Tibshirani R. Regularization paths for generalized linear models via coordinate descent. J Stat Softw. 2010;33(1):1-22.

\section{Publisher's Note}

Springer Nature remains neutral with regard to jurisdictional claims in published maps and institutional affiliations.
Ready to submit your research? Choose BMC and benefit from:

- fast, convenient online submission

- thorough peer review by experienced researchers in your field

- rapid publication on acceptance

- support for research data, including large and complex data types

- gold Open Access which fosters wider collaboration and increased citations

- maximum visibility for your research: over $100 \mathrm{M}$ website views per year

At BMC, research is always in progress.

Learn more biomedcentral.com/submissions 